\section{Cureus}

Received 11/30/2017

Review began 12/01/2017

Review ended 03/10/2018

Published 04/08/2018

\section{(c) Copyright 2018}

Le et al. This is an open access article distributed under the terms of the Creative Commons Attribution License CC-BY 3.0., which permits unrestricted use, distribution, and reproduction in any medium, provided the original author and source are credited.

\title{
Pruritic Rash in a Patient with Hodgkin's Lymphoma
}

\author{
Audrey Le ${ }^{1}$, Dimitrios Farmakiotis ${ }^{2}$, John L. Reagan ${ }^{3}$ \\ 1. Internal Medicine, Rhode Island Hospital, Alpert Medical School of Brown University 2. Infectious \\ Diseases, Rhode Island Hospital, Warren Alpert Medical School of Brown University 3. Oncology- \\ Hematology, Rhode Island Hospital, Warren Alpert Medical School of Brown University
}

$\square$ Corresponding author: Audrey Le, audrey.le@lifespan.org

Disclosures can be found in Additional Information at the end of the article

\section{Abstract}

Bleomycin-induced flagellate erythema (FE), a skin finding associated with cutaneous deposition of bleomycin, is so called due to its characteristic pattern of whip-like, linear streaks. As bleomycin use in standard chemotherapeutic regimens has decreased, the clinical diagnosis has become increasingly rare. The authors present a case of a 43 -year-old female patient with Hodgkin's lymphoma on her first cycle of adriamycin, bleomycin, vinblastine, and dacarbazine (ABVD) treatment, who subsequently developed a diffuse rash classic for FE. This benign condition is important to recognize to avoid potentially unnecessary and harmful treatment for other dermatologic diagnoses for which it may be mistaken. In severe cases of FE, discontinuation of bleomycin should be considered.

Categories: Dermatology, Allergy/Immunology, Oncology

Keywords: bleomycin-induced flagellate erythema, flagellate dermatosis, abvd, bleomycin, pruritic rash

\section{Introduction}

Bleomycin is a sulphur-containing antimicrobial derived from Streptomyces verticillus used in standard chemotherapy for Hodgkin's lymphoma, germ cell tumors, Kaposi sarcoma, and pleurodesis for malignant pleural effusions [1-4]. Bleomycin penetrates all tissues, but is quickly inactivated by bleomycin hydrolase, which is absent in the lungs and skin; thus its toxic manifestations occur predominantly in these two organs $[1,3,5]$. Studies show that $60-70 \%$ is excreted in the urine [3], hence patients with renal dysfunction may be at higher risk for toxicity $[2,6]$. Bleomycin-induced flagellate erythema (FE), associated with skin deposition of bleomycin, is named after the initial eruption of erythematous linear streaks that appear 'whiplike' $[3,7]$.

We present a case of a female patient with Hodgkin's lymphoma who presented with classic findings for FE after initiation of chemotherapy.

\section{Case Presentation}

A 43-year-old woman with classic Hodgkin's lymphoma (HL) presented on Day 7 of her first cycle of adriamycin, bleomycin, vinblastine, and dacarbazine (ABVD) with a diffuse, pruritic maculopapular and nodular rash, which had started from her knuckles and progressed to the torso and lower extremities (Figure 1). 


\section{Cureus}
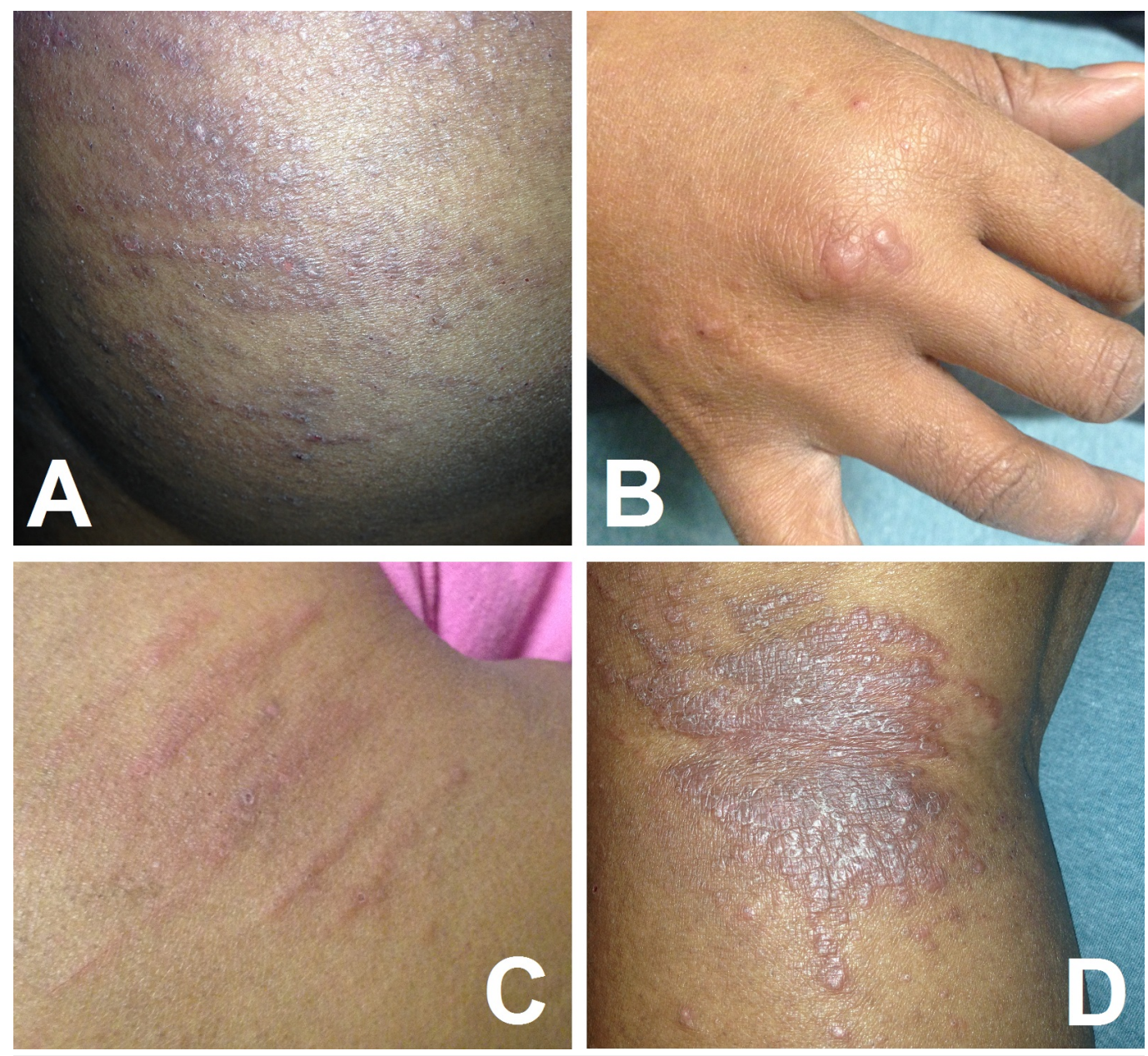

\section{FIGURE 1: Bleomycin-induced flagellate erythema}

The pruritic maculopapular rash and nodules, on the torso $(A)$, upper $(B)$ and lower $(C, D)$ extremities, with which our patient presented after her first cycle of ABVD, classic for bleomycininduced flagellate erythema. ABVD - adriamycin, bleomycin, vinblastine, and dacarbazine.

The presence of her diffuse rash along with a pulmonary cavitary lesion prompted infectious workup. She had a positive urine histoplasma antigen (Quest diagnostics, NJ, USA) and was admitted to the hospital for treatment of presumed histoplasmosis with intravenous amphotericin-B. Skin biopsy was performed and histopathology showed inflammatory changes suggestive of drug reaction. Repeat histoplasma urinary antigen was negative, and computerized tomography of the chest showed significant decrease in the size of the pulmonary lesion after one ABVD cycle, consistent with HL as the cause of her pulmonary cavitary lesion. She was discharged with a diagnoses of bleomycin-induced flagellate erythema and falsely positive urine histoplasma antigen. Her skin lesions completely resolved over the next two weeks with symptomatic treatment (two antihistamines for intense pruritus, triamcinolone $0.1 \%$ cream, and petrolatum-based skin protectant).

\section{Discussion}

FE has become increasingly rare as bleomycin use has decreased; however, it is important to note that there have been reported cases of FE amongst other patient populations, particularly those with dermatomyositis and adult-onset Still's disease, as well as in individuals after shiitake mushroom ingestion [6-8]. Peplomycin, a bleomycin analogue used in the treatment of prostate cancer, breast cancer, and HL, can also cause FE [7, 8]. It is important to rule out other 
causes of parallel, streak-like skin lesions, such as Koebner phenomenon due to inflammatory, infectious, or self-inflicted processes, or photodermatitis [7]. Bleomycin-induced FE has variable onset, occurring anywhere between several hours to two months after drug administration [9]. There is no clear association between FE and the route of drug administration, dose, or type of underlying disease being treated [6]. Lesions most frequently appear on the torso, extremities, and nape of the neck, but they can present on the face and scalp as well $[4,5,7,9]$. FE requires symptom relief with antihistamines and corticosteroids (topical or oral). Discontinuation of bleomycin is generally reserved for severe cases of skin toxicity, since in an open-label, randomized, multicenter trial comparing ABVD with regimens omitting bleomycin, dacarbazine, or both, in early-stage favorable Hodgkin's lymphoma, there was no significant difference in survival. However, patients treated without bleomycin had higher rates of relapse and worse control of tumor burden [10].

Given the severity of symptoms in our patient, she resumed AVD chemotherapy (without bleomycin), followed by salvage brentuximab and bendamustine for primary refractory disease, and subsequent autologous stem cell transplantation with post-transplant brentuximab. She is disease-free and doing well two years after FE diagnosis, 18 months after transplant.

\section{Conclusions}

Clinicians should be aware of this uncommon, yet benign complication, to avoid unnecessary workup and potentially harmful empiric treatment for other causes of skin lesions in immunocompromised patients given the broad differential diagnosis. In severe cases of FE, discontinuation of bleomycin should be considered.

\section{Additional Information \\ Disclosures}

Human subjects: Consent was obtained by all participants in this study. Conflicts of interest: In compliance with the ICMJE uniform disclosure form, all authors declare the following:

Payment/services info: Dr. John Reagan receives funding for development of educational presentations from Teva Pharmaceuticals and serves on a speaker bureau for Alexion Pharmaceuticals. Dr. Dimitrios Farmakiotis has received research support from Astellas and consultation fees from Viracor. Dr. Audrey Le has no potential conflicts of interest or sources of research funding. . Financial relationships: John L. Reagan declare(s) Funding from Teva Pharmaceuticals. Dr. Reagan receives funding for development of educational presentations from this company. . John L. Reagan declare(s) personal fees from Alexion Pharmaceuticals. Dr. Reagan serves on a speaker bureau for Alexion Pharmaceuticals. Dimitrios Farmakiotis declare(s) personal fees from Viracor. Dr. Dimitrios Farmakiotis has received consultation fees from Viracor. Dimitrios Farmakiotis declare(s) Research funding from Astellas. Dr. Dimitrios Farmakiotis has received research support from Astellas. . Other relationships: All authors have declared that there are no other relationships or activities that could appear to have influenced the submitted work.

\section{References}

1. Dantzig PI: Immunosuppressive and cytotoxic drugs in dermatology . Arch Dermatol. 1974, 110:393-406. 10.1001/archderm.1974.01630090031007

2. Yamamoto T: Bleomycin and the skin. Br J Dermatol. 2006, 155:869-875. 10.1111/j.13652133.2006.07474.x

3. Fyfe A, McKay P: Toxicities associated with bleomycin. J R Coll Physicians Edinb. 2010, 40:213-215. 10.4997/JRCPE.2010.306

4. Chen Y-B, Rahemtullah A, Breeden E, Hochberg EP: Bleomycin-induced flagellate erythema. J Clin Oncol. 2007, 25:898-900. 10.1200/JCO.2006.09.7691 


\section{Cureus}

5. Simpson R, Da Forno P, Nagarajan C, Harman KE: A pruritic rash in a patient with Hodgkin lymphoma. Bleomycin-induced flagellate dermatosis. Clin Exp Dermatol. 2011, 36:680-682. 10.1111/j.1365-2230.2010.03995.x

6. Vennepureddy A, Siddique M, Odaimi M, Terjanian T: Bleomycin-induced flagellate erythema in a patient with Hodgkin's lymphoma - a case report and review of literature. J Oncol Pharm Pract. 2016, 22:556-560. 10.1177/1078155215580468

7. Mendonca FM, Marquez-Garcia A, Mendez-Abad C, Rodriguez-Pichardo A, Perea-Cejudo M, Rios Martin JJ, Camacho FM: Flagellate dermatitis and flagellate erythema: report of 4 cases . Int J Dermatol. 2017, 56:461-463. 10.1111/ijd.13523

8. Yamamoto T, Nishioka K: Flagellate erythema. Int J Dermatol. 2005, 45:627-631. 10.1111/j.1365-4632.2005.02647.x

9. Biswas A, Chaudhari P, Sharma P, Singh L, Julka PK, Sethuraman G: Bleomycin induced flagellate erythema: revisiting a unique complication. J Cancer Res Ther. 2013, 9:500-503. 10.4103/0973-1482.119358

10. Behringer K, Goergen H, Hitz F, et al.: Omission of dacarbazine or bleomycin, or both, from the ABVD regimen in treatment of early-stage favourable Hodgkin's lymphoma (GHSG HD13): an open-label, randomised, non-inferiority trial. Lancet. 2015, 385:1418-1427.

10.1016/S0140-6736(14)61469-0 